\title{
Cellular and Biochemical Changes in the Aging Mouse Immune System
}

\author{
Richard A. Miller, M.D., Ph.D.
}

My perspective is different from that of Dr. Weksler. I see age-related loss of responsiveness as a cardinal feature of immunosenescence, one that puts elderly people at risk of infection and perhaps neoplasia. It is possible that age-associated increases in the activity of some kinds of immune cells, which Dr. Weksler has emphasized, contribute to this decline in protective immunity, although this hypothesis needs a good deal of further investigation. I do not see autoimmunity as a major threat to the elderly or an important component of immunosenescence, although the jury is still out on this, and clearly investigators of autoreactivity have the potential for teaching us a lot about how the aged immune system works.

Thus in my view one of the most pressing challenges for immunogerontologists is to figure out why the immune system generates fewer antibodies to specific antigens, fewer cytotoxic $\mathrm{T}$ cell effectors, and less protection against potential infections and pathologic agents in elderly humans and old mice. I will call this deficiency of function, and I think that some of the cross-wiring that Dr. Weksler and others have pointed out may give us a way of exploring it anew. I am not convinced by arguments to date that have suggested an important pathological implication for autoimmunity. Some autoimmune phenomena are demonstrated in elderly mice and humans, but their pathological implications are still mysterious; more work needs to be done to understand the implications for health and disease.

The cellular and molecular basis for the agerelated decline in immune responsiveness can be enumerated in several ways. First, there is an agerelated change in $\mathrm{T}$ cell subsets. Figure 1 shows that there is an age-associated accumulation of memory (Pgp- $\left.1^{\text {hi }}\right) \mathrm{T}$ cells. There is a decrease with age in the number of virgin $T$ lymphocytes resulting in a relatively greater proportion of memory as com-

Dr. Miller is a Professor of Pathology and the Associate Director for Research at the Geriatric Center, University of Michigan, Ann Arbor, MI 48109, USA. pared to virgin $T$ cells. This result has been found in many laboratories in both mice and humans. When one uses $\mathrm{CD} 44^{+}$, a marker of memory cells, one finds nearly triple the number of memory cells in the spleen, lymph nodes, or blood of old compared to young mice.

Second, is this change in $T$ cell subsets important and do these shifts contribute to the age-associated changes in cytokine production? Kevin Flurkey and his colleagues' use a limiting dilution, cellcounting assay. Figure 2 shows that $T$ cells from young animals (about 55\%) could make interleukin2 (IL-2) when they were stimulated with concanavalin (Con) A. Virgin cells purified from the T cells can make IL-2 and IL-3. In this kind of assay the purified memory lymphocytes do not make IL-2 or IL-3, regardless of whether they are stimulated with Con A (Figure 2) or with staphylococcal enterotoxin (SEB, known to trigger the $\mathrm{T}$ cell receptor) (Figure 3). Essentially all the responder cells in young and old mice are of the virgin type. The accumulation of this memory cell population accounts for the age-associated loss in the fraction of cells in an unsorted T cell population that can make IL- 2 and IL-3.

In addition to measuring lymphokine production, we have looked at the ability of cells to respond to IL-2. Figure 4 shows results from a limiting dilution (LD) assay to identify the precursors of proliferating and cytotoxic $\mathrm{T}$ lymphocytes. There is an age-related loss in the number of both proliferating and cytotoxic $\mathrm{T}$ cell precursors. This is an IL-2 driven reaction, as shown in Figure 4. In young or old mice, naive cells respond well while the memory cells do not respond as well. The age-associated increase in this memory cell fraction largely accounts for the age-related loss in the number of cells that proliferate or generate cytotoxic effectors in this IL-2-driven reaction. A more subtle point is that the function of memory cells also decreases with age.

The accumulation of memory cells largely accounts for the age-related loss of Con A respon- 


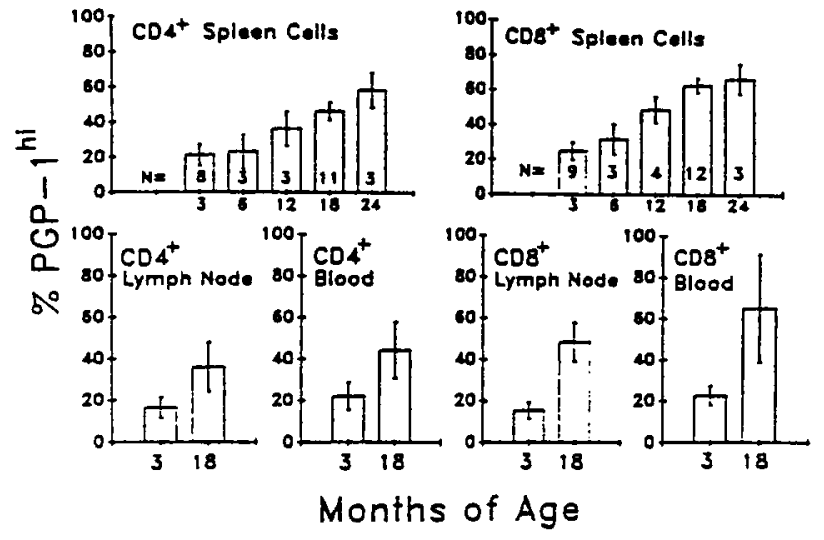

Figure 1. Age-related increase in the proportion of Pgp$1^{\text {hi }}$ cells in spleen, lymph node, and blood. Separate analyses were performed for $\mathrm{CD}^{+}{ }^{+}$and for $\mathrm{CD} 8^{+}$cells. For the spleen cells, $n$ values (shown within the bars) refer to the number of individual mice tested. $n=5$ for each bar in the blood and lymph node experiments. The error bars refer to $95 \%$ confidence intervals on the mean. Reprinted with permission from Lerner et al. ${ }^{5}$

siveness, of IL-2 and IL-3 production, and of IL-2responsive cells. We have some hints as to the molecular basis. We studied two aspects of early signal transduction. Each panel of Figure 5 is a histogram of 10,000 young or 10,000 old unstimulated $T$ cells from our calcium assay. The horizontal axis is proportional to the calcium concentration in individual cells. Time 0 shows that the calcium level is almost identical in young and old cells prior to stimulation. When cells are stimulated with Con A, however, after 5 minutes (the peak of the calcium response) $70-75 \%$ of the $T$ cells from young mice have generated a calcium signal. In old mice there is also a calcium response, and the kinetics are the same as those of young mice, but the number of cells that generate a calcium signal has declined by a factor of two. Thus, there is an age-related loss in the fraction of cells that can generate a calcium signal.

We have also looked at other early signal transduction molecules, for example protein kinases. In Figure 6 we have taken T cells, stimulated them for 2,5 , or 10 minutes with anti-CD3 and then, by western blot analysis method, estimated the amount of tyrosine-specific phosphorylation of three substrates. The top panel shows a substrate with a molecular weight of $120 \mathrm{kDa}$, the middle panel 80 , and the lower panel $40 \mathrm{kDa}$. These three species are stimulated by Con A or anti-CD3. Each point represents an average of 9-11 young and 9-11 old mice. For all three substrates, and for all three time points, the young mice generate more tyrosine-specific protein kinase than that generated by old mice. So calcium signals and kinase specific signals decline with advancing age.

Can we explain these signal changes on the ba-

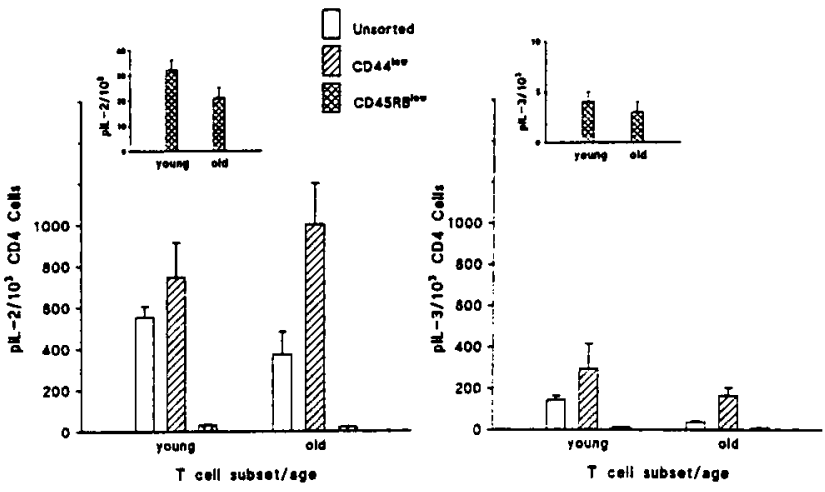

Figure 2. Preferential activation of naive $T$ cells by Con A. pIL-2 (left panel) and pIL-3 values (right panel) represent means (+SEM) of three experiments, each involving splenocyte samples from one young (3-6 month) and one old (19-20 month) female mouse and are expressed per $10^{3} \mathrm{CD}^{+}$cells. The frequencies of plL-2 and pIL-3 were higher among CD $44^{\text {lo }}$ cells $(p<0.01$ for pIL-2 cells and $p<0.001$ ) compared to unsorted controls (ANOVA followed by planned comparisons of cells subsets, age groups combined, using log-transformed data). Data for unsorted cells from young and old mice are provided for comparison to other cell subsets. Inserts show precursor frequencies of the CD45RB ${ }^{\text {lo }}$ cells on an expanded scale. Reprinted with permission from Flurkey et al.'

sis of the cellular changes, related to a shift to produce memory lymphocytes? Does this account for early changes in the inability of cells to respond to stimulating agents? The answer is "yes" for calcium and "yes" for the tyrosine-specific kinases. Philosophe's work ${ }^{2}$ shows the calcium data (Figure 7). The vertical axis is $\mathrm{CD}_{4} 4^{+}$or P-GP-1+ ${ }^{+}$, everything

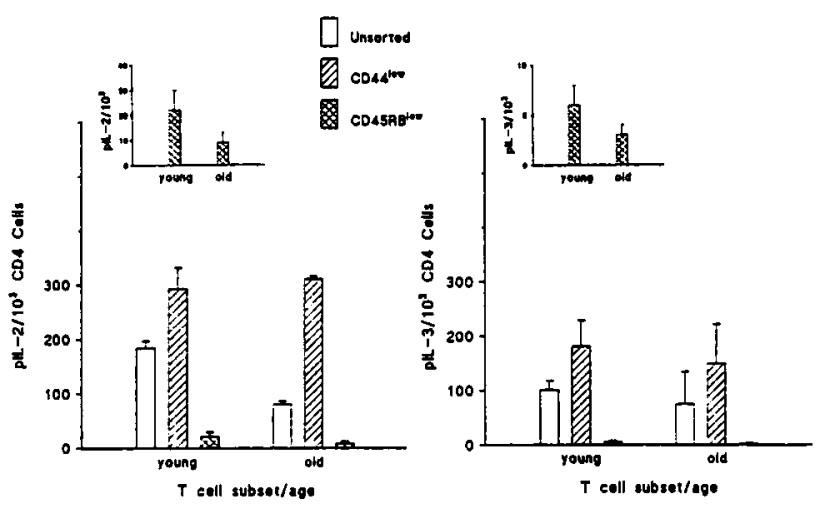

Figure 3. Preferential activation of naive $T$ cells by SEB. pIL-2 (left panel) and pIL-3 values (right panel) represent means (+SEM) of three experiments, each involving splenocyte samples from one young (3-6 month) and one old (19-20 month) female mouse, expressed per $10^{3}$ $\mathrm{CD}^{+}$cells. The frequencies of pIL-2 and pIL-3 were enhanced in the CD44 $4^{\text {lo }}$ subset $(p<0.05)$ and diminished in the CD45RB ${ }^{10}$ subset $(p<0.001)$ compared to controls (ANOVA, planned comparisons: log-transformed data). Reprinted with permission from Flurkey et al.' 

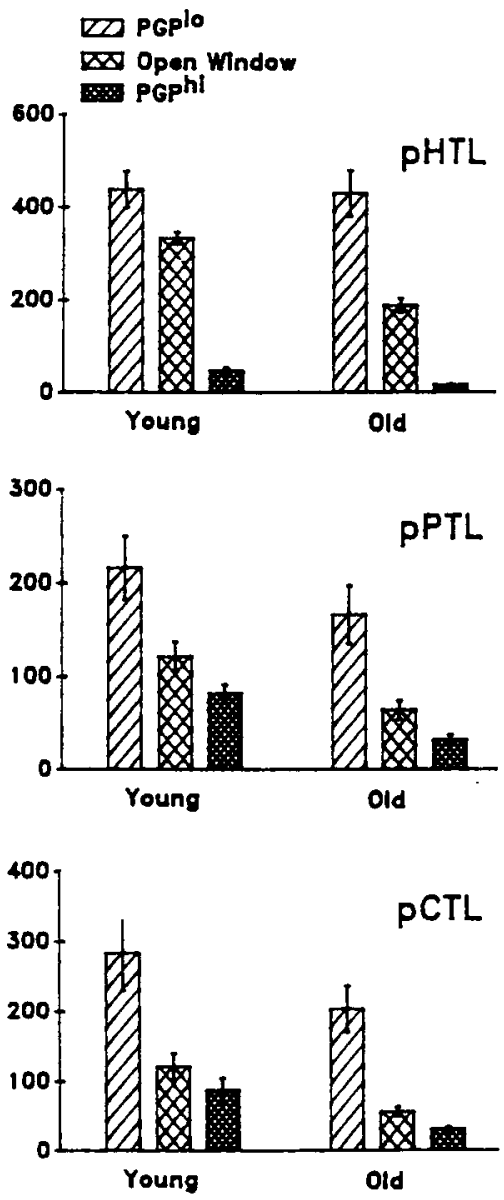

Figure 4. Responder cell frequencies among splenic $T$

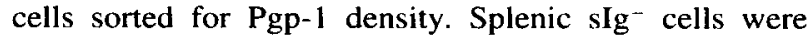
stained for Pgp-1, gated by forward- and site-scatter signals, and sorted into Pgp- $1^{\text {hi }}$ (upper quartile) and Pgp- $1^{\text {lo }}$ (lower quartile) populations. Sorted cells, together with a mock-sorted (open window) control population, were then tested by LD to estimate their frequency of Con A-induced responsive helper (pHTL), proliferator (pPTL) and cytoxic (pCTL) cells. Values shown are means \pm SEM for $n=9$ experiments, each involving one young and one old mouse. Reprinted with permission from Lerner et al. $^{5}$

above the dotted line is a memory cell, and everything below is a virgin cell. Unstimulated memory and virgin cells have the same calcium levels. Once stimulated with Con A or antibody to the CD3 complex, or even ionomycin, which bypasses the signaltransducing mechanisms completely, cells show the same pattern of response. Memory cells are quite resistant to the increase in calcium signals; virgin cells generate quite a nice calcium signal to Con A, anti-CD3, or ionomycin. The ionomycin response, in particular, suggests that there might be an intrinsic resistance of memory cells to increases in their calcium level. This might be mediated through a pump that pumps calcium out of the cell and back into the medium.
We have also used a cell-sorter to isolate cells that generate a calcium signal in both young and old mice. Figure 8 represents cells that do $\left(\mathrm{Ca}^{\mathrm{R}}\right)$ or do not $\left(\mathrm{Ca}^{\mathrm{NR}}\right)$ generate a calcium signal after they were exposed to ionomycin. Most of the $\mathrm{Ca}^{\mathrm{R}}$ cells could proliferate, make IL-2, and turn into cytotoxic cells. Cells that do not generate a calcium signal are also functionally incompetent. That is true for young and old mice. If the calcium signal is generated, it largely accounts for the ability of cells to proliferate in response to IL-2, make IL-2 or IL-3, or generate cytotoxic clones. In the case of calcium, the cellular changes probably can explain the biochemical changes. This is also true for changes in tyrosine-specific protein kinases. Figure 9 shows the effect of age on the phosphorylation of three antiCD3-inducible phosphotyrosine-containing proteins using results from western blots. Unfractionated $\mathrm{CD} 4^{+} \mathrm{T}$ cells do better than the memory cell subsets of the $\mathrm{CD} 4^{+} \mathrm{T}$ cells. When memory cells have been isolated, you have cells that do not generate strong phosphorylation of any of these responsive proteins.

The age-associated increase in memory cells also accounts for the shift toward a decline in tyrosine-specific kinase phosphorylation of these three substrates. The major weakness in these data is that we do not yet know the function of these substrates or kinases.

Finally, I would like to mention two new investigations. First, we are subdividing the virgin subsets or the memory subsets even further in functional ways. Jacek Witkowski has taken advantage of a property of the dye called rhodamine 123 (R123). ${ }^{3}$ R123 stains young and old T cells equally well, but if the stained cells sit for 30 minutes the old $T$ cells pump the rhodamine out; the young $T$ cells do not. We believe this phenomenon is due to P-glycoprotein, an ATP-dependent plasma membrane pump that mediates multiple-drug resistance. This pump is present in normal cells, including normal lymphocytes of old mice. The reason it is worth presenting in this context is that it subdivides the virgin and memory $T$ cell pools. Figure 10 shows memory and naive cells from mice of different ages; the memory cells (CD44 $\left.{ }^{\text {hi }}\right)$ are in the upper half and the virgin cells $\left(\mathrm{CD} 44^{10}\right)$ are shown in the lower half. The R123-high population includes both memory and virgin cells. Even in the young mice, there is a subset of memory cells that can pump out rhodamine because they have considerable P-glycoprotein. With aging, there are more memory cells; however, in this context, the critical issue is that in old mice, more than $56 \%$ of memory cells have a lot of P-glycoprotein and pump the rhodamine.

We wondered whether this change has any functional implication. Is there anything memory cells 


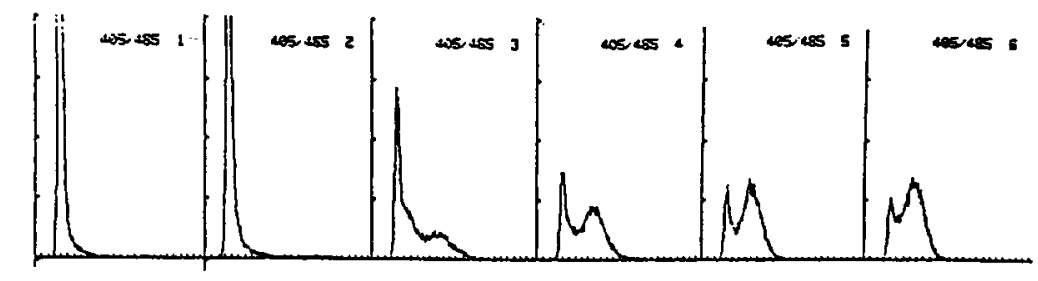

Young
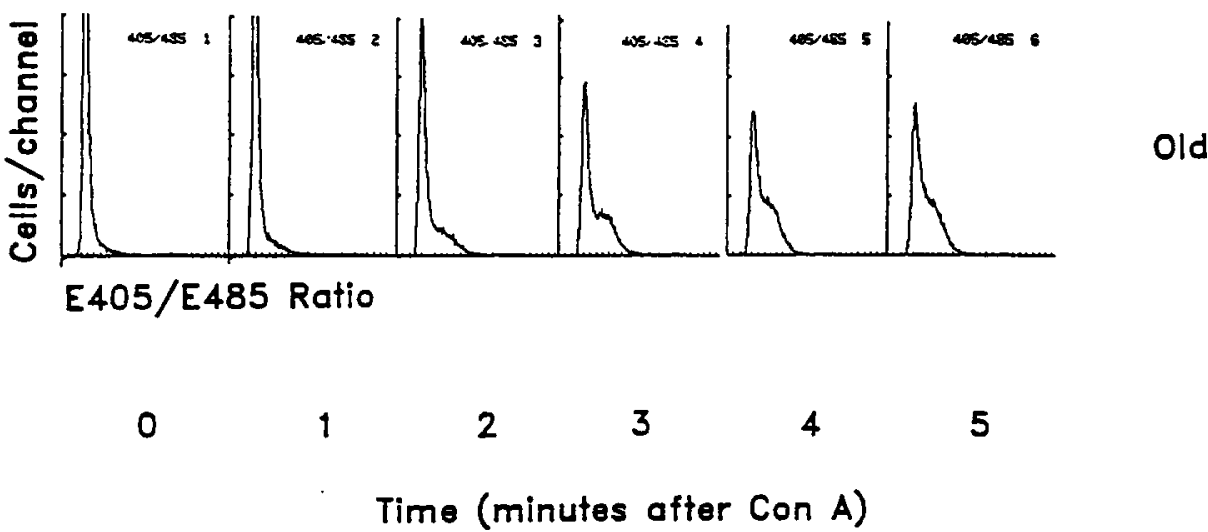

Figure 5. Flow cytometric analysis of T-cell activation. One pair of analyses is shown, representative of eight young and seven old mice tested. Each histogram presents an analysis of 10,000 cells; the vertical axis gives the number of $T$ cells with the fluorescence ratio $\left(\mathrm{E} 405 / \mathrm{E} 485\right.$, a measure of $\mathrm{Ca}_{\mathrm{i}}$ ) corresponding to each channel on the horizontal axis. Channels on the right correspond to increasing levels of $\mathrm{Ca}_{\mathrm{i}}$. Each set of six panels (young $\mathrm{T}$ cells at the top: old $\mathrm{T}$ cells at the bottom) depicts cells sampled at 1 -minute intervals after addition of $10 \mu \mathrm{g} / \mathrm{mL}$ Con A to a suspension of 2 $\times 10^{6} \mathrm{~T}$ cells in $1 \mathrm{~mL}$ of Krebs Ringer Phosphate at $37^{\circ} \mathrm{C}$. Reprinted with permission from Miller et al. ${ }^{6}$

cannot do in old mice that could be attributed to this shift? To investigate this, we looked at IL-4 production in a novel assay. In short-term assays the reported results are controversial, with some showing increases in IL-4 with advancing age, while others have reported decreases in production. We used different assay conditions to stimulate $T$ cells with anti-CD3 for 2 days, then adding IL-2 to the cultures, since IL- 2 has been shown to be important in IL-4 production. Figure 11 shows that IL-4 production 3, 6, or 9 days after the addition of IL-2 was higher in young mice than in old mice. Thus, under this assay condition, aging seems to lead to a decline in the response to IL-2 as measured by IL-4 production. We found, as many others have found previously, that the memory cell population is responsible for this. Memory cells from old mice are plentiful but they are functionally deficient in terms of IL-2-driven IL-4 production. So we asked what if we looked at this after subdividing the memory cells using the rhodamine exclusion assay. We found that the rhodamine-high population, the subset predominant in young mice, makes essentially all the IL-4. ${ }^{3}$ We started with $\mathrm{CD} 4^{+}$memory cells so we were not worried about contributions from $\mathrm{CD} 8^{+}$or virgin cells-just the memory $\mathrm{CD}^{+}$cells. Of that subset, it is only the rhodamine-high population that can make IL-4. The rhodamine-low population, which increases with age, is unable to make IL-4.

Looking at other antigens, other lymphokines, and other stimuli establishes the principle that the rhodamine dichotomy, which is age sensitive, has at least one functional implication and possibly others we now need to study. Even the rhodamine-high population in old mice does not adequately make IL-4. It is better than the rhodamine-low population, but there is a defect in IL-4 production that cannot simply be explained by rhodamine sensitivity.

The question remains whether all of these cellular or molecular changes have implications for health. We have done a longitudinal study in mice, and we are about halfway through it. I will present the initial results. We tested mice at 6 months of age for several age-sensitive aspects of immune function to determine whether mice that looked immunologically old at 6 months of age were more susceptible to cancer. We used a genetically heterogeneous population rather than inbred mice, because inbred animals tend to die of the same pathology. A four-way cross-breeding scheme insured that we had a population in which no two mice were genetically identical. We then measured immune function and then let these mice die of natural causes. Five of the group of 40 died before their 18month birthday; all died of some form of cancer. 

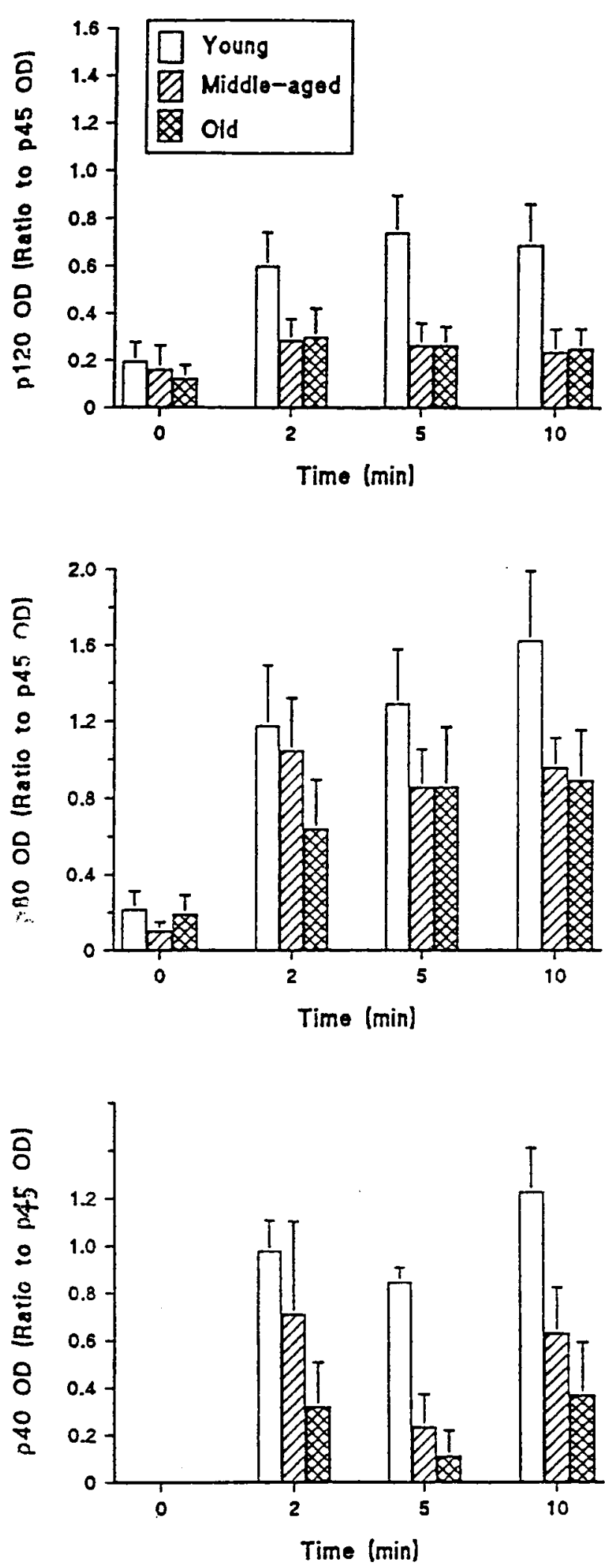

Figure 6. Age-related decline in tyrosine phosphorylation of three substrates in response to $\alpha \mathrm{CD} 3$ antibody. Values are means \pm SEM from 7-10 experiments per time point for $\mathrm{p} 120$ and $\mathrm{p} 80$, and 4-6 experiments per time point for $\mathrm{p} 40$. Reprinted with permission from Shi and Miller. ${ }^{7}$

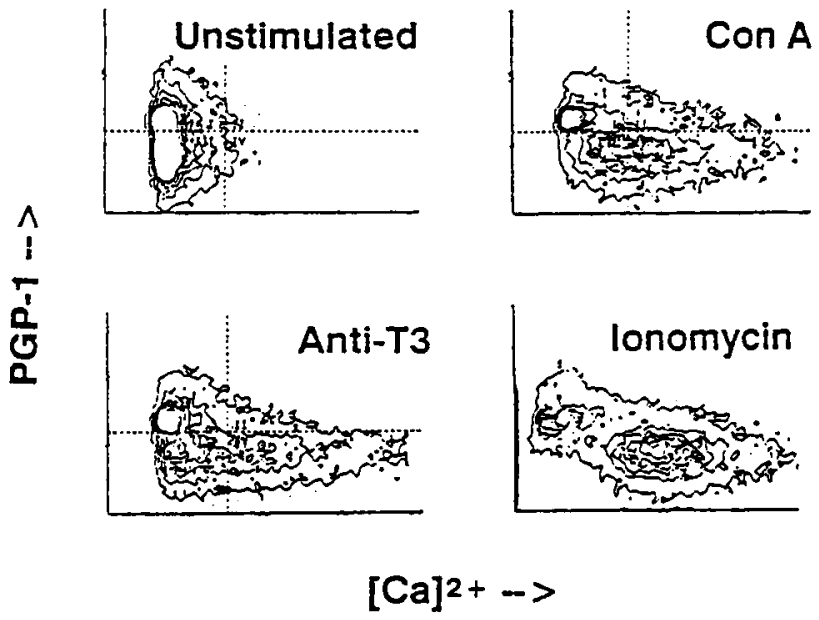

Figure 7. Differential responses of $\mathrm{Pgp}-1^{+}$and $\mathrm{Pgp}-1^{-} \mathrm{T}$ cells to activating agents. The distribution of calcium concentrations was measured in $T$ cells (from a young mouse) prior to stimulation, or after exposure to Con $\mathrm{A}$ (10 $\mu \mathrm{g} / \mathrm{mL}$ ), $\alpha \mathrm{CD} 3$ antibody (1:10,000 dilution of ascites; cross-linked with $5 \mu \mathrm{g} / \mathrm{mL}$ rabbit anti-mouse $\mathrm{Ig}$ ), or ionomycin $(20 \mathrm{ng} / \mathrm{mL})$. T cells were labeled with indo-1 and with fluorescein-conjugated anti-Pgp-1 antibody, and samples of 20,000 cells were examined by flow cytometry either 3 minutes ( $\alpha \mathrm{CD} 3$, ionomycin) or 4 minutes (Con A) after activation. The vertical axis shows log green fluorescence as a measure of Pgp-1 staining; the horizontal dashed line indicates the threshold used to distinguish Pgp- $1^{+}$from Pgp- $1^{-}$T cells. The horizontal axis represents the ratio of indo- 1 emission at $405 \mathrm{~nm}$ divided by emission at 480 , as a measure of intracellular free calcium ion concentration. Ninety percent of the unstimulated cells have $[\mathrm{Ca}]_{i}$ values less than that indicated by the vertical dashed line (calcium threshold). The calcium scale for the ionomycin treatment differs from that of the other three panels, since even the least-responsive cells increase $[\mathrm{Ca}]_{i}$ to some extent when treated with ionomycin. Reprinted with permission from Philosophe and Miller. $^{2}$
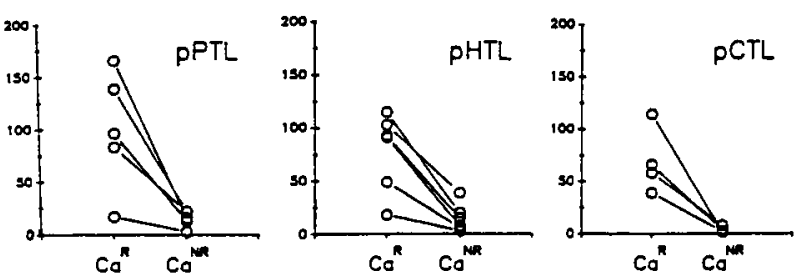

Figure 8. LD analyses of cells from young donors sorted into calcium responder $\left(\mathrm{Ca}^{\mathrm{R}}\right)$ and calcium non-responder $\left(\mathrm{Ca}^{\mathrm{NR}}\right)$ pools after stimulation with $\mathrm{Con} \mathrm{A}$. Each line connects points representing frequency determinations from a single experiment. Results are shown for assays of Con A-responsive proliferating (pPTL), IL-2-secreting (pHTL), and cytoxic (pCTL) T cells. Reprinted with permission from Philosophe and Miller. ${ }^{8}$ 

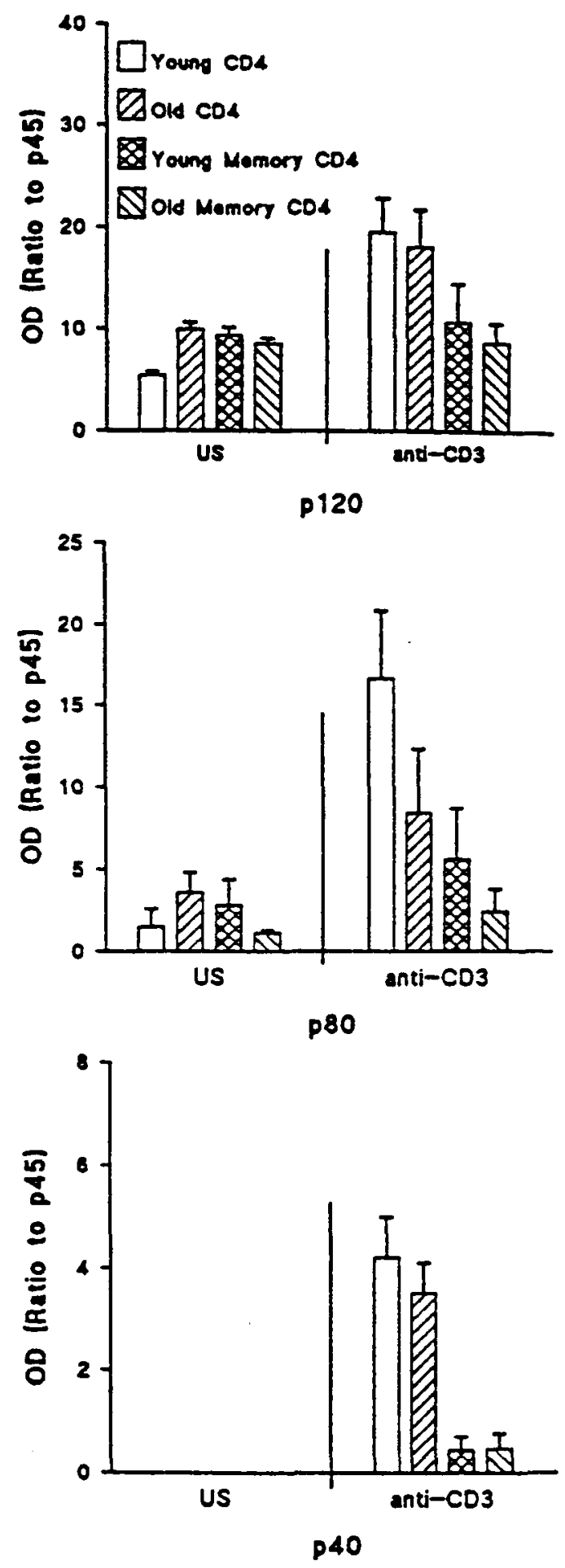

Figure 9. Effect of anti-CD3 on levels of tyrosine-specific protein phosphorylation in unfractionated CD4 and CD4 memory $T$ cells from old and young mice. Values are mean \pm SEM from four experiments. US, unstimulated. Reprinted with permission from Shi and Miller. ${ }^{9}$

There were four different forms in the population, but all the deaths were from some aspect of neoplastic disease. There was a statistically significant trend for the mice that died early to have aged im-

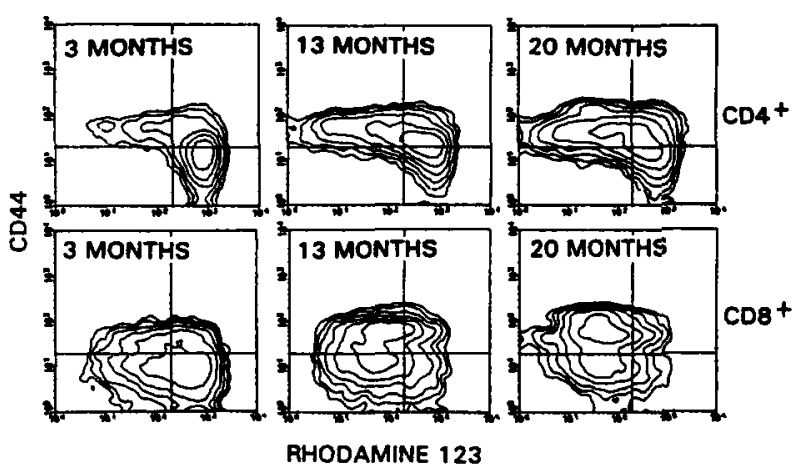

Figure 10. R123 staining distributions within naive $\left(\mathrm{CD} 44^{\mathrm{lo}}\right)$ and memory $\left(\mathrm{CD} 44^{\mathrm{hi}}\right) \mathrm{T}$ cell subsets in young, middle-aged, and old mice. $T$ cells stained with R123, anti-CD4 (top panels) or anti-CD8 (bottom panels), and anti-CD44. The CD4 (or CD8) signal was used to exclude $\mathrm{CD}^{-}{ }^{-}$or $\mathrm{CD}^{-}$from the analysis. The horizontal line shows the threshold used to discriminate CD44 from CD $44^{10}$ cells, and the vertical line shows the criterion used for R123 discrimination. Reprinted with permission from Witkowski and Miller. ${ }^{10}$

mune systems. The $\mathrm{CD} 44^{+}$memory population of the $\mathrm{CD}^{+}$cells was high at 6 months in those animals destined to die young. The $\mathrm{CD} 44^{+}$population of the $\mathrm{CD}^{+}$cells, memory killer cells, was also statistically significantly higher in these animals.

We also looked at virgin cells. Our prediction

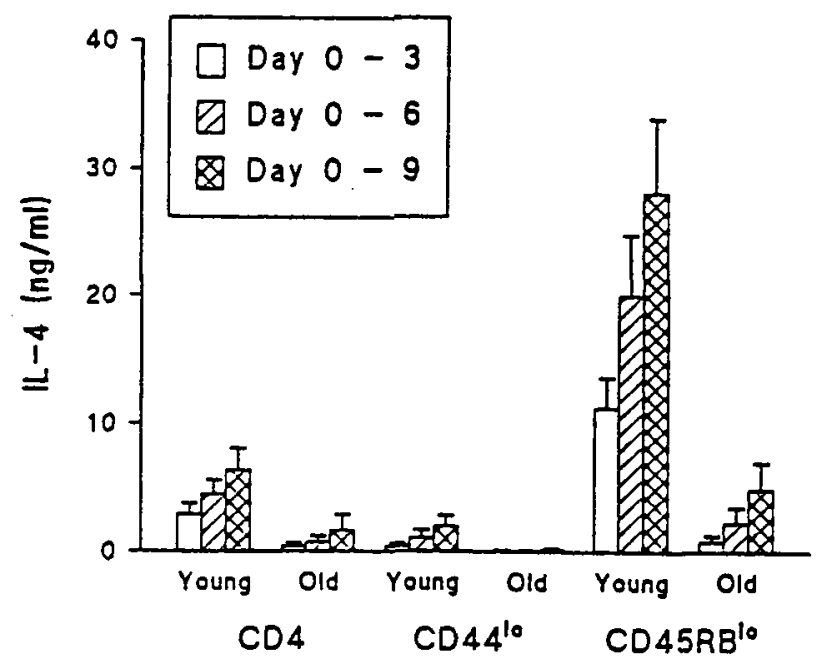

Figure 11. IL-4 secretion by unfractionated, naive, and memory CD4 $\mathrm{T}$ cells from young and old mice in 9-day cultures. Each $0.2-\mathrm{mL}$ well contains $10^{4} \mathrm{~T}$ cells of the indicated subset from young or old donors, as well as $10^{4}$ young resident peritoneal cells (RPC) as accessory cells. Cells were preactivated by incubation with anti-CD3 antibody for 2 days and then further incubated with IL-2 plus. anti-CD3 for the indicated expansion time. The level of IL-4 produced during each indicated culture interval is plotted as mean \pm SEM for $n=7$ independent experiments. Reprinted with permission from $\mathrm{Li}$ and Miller." 
was that they would be lower in mice destined to die because virgin cells decline with age. That is what we found. The five mice that died at 18 months had, at 6 months of age, fewer virgin cells than their counterparts that were going to live beyond their 18-month birthday. ${ }^{4}$

We also found that functional indices of immune response correlated with the development of neoplastic diseases. For example, of the fraction of blood cells that could respond to Con A and IL-2 by proliferating, those that had low levels of Con A- and IL-2-stimulated proliferation were also in the mice destined to die of cancer before their 18month birthday. We hope that what we are measuring here is an association between $\mathrm{T}$-cell status and tumor development as indicators of underlying aging rate. We would have also been happy if instead we had proven that aging of the immune system predisposes an individual to tumor development. It is an open and hotly contested issue as to whether the immune decline with age does or does not leave elderly people more susceptible to cancer. One may ask whether animals with an aging immune system get cancer more quickly than younger animals. It is possible that an early phase of neoplastic transformation in the 6-month-old mice led secondarily to a change in their immune function. We picked up that change in immune function, and a year later animals with impaired immune functions died. However, four forms of cancer were found in the population, and these are the type of tumors that are generally not detectable a year before death, but we still have not ruled out this possibility.

What I have tried to convey is that there is a meaningful way to talk about immune deficiency in the elderly. Changes in the cellular composition of the immune system are detectable by memory cell markers, virgin cell markers, and the newer P-glycoprotein marker. These age-associated changes account for some, but not all, of the functional changes, some lymphokine responses, and some lymphokine-production responses. In our preliminary longitudinal study, these markers allowed us to identify, early in life, mice that are more prone to cancer or early death. These correlations could represent some link to an underlying aging rate or could represent an ability of the healthy immune system to protect animals from cancer.

\section{Discussion}

\section{Weksler:}

Despite Dr. Miller's apologia at the beginning of his talk, I feel that all his data are compatible with the state of dysregulation of the immune system. With regard to whether autoantibodies are good or bad, clearly that is a very difficult subject. One could say that they are associated with bad things, but that might just be coincidence and this has always been the question with autoimmune disease. Just because two things are linked does not mean that they are causally related. However, I think we are increasingly recognizing that certain autoantibodies can, in fact, transmit disease, like myasthenia gravis. So in certain cases we are making a stronger argument that the association is not just an association. Certainly autoimmune "disease" - if you want to consider it as such —of aging is a good deal more subtle than the autoimmune diseases we currently recognize. It would be increasingly difficult to prove causality. I have tried to show one type of autoantibody, auto-anti-idiotypic antibody is associated with a poor response to normal antigens.

With regard to Dr. Miller's provocative studies in neoplastic disease, the one question I have is that since four out of five malignancies were lymphoid, do you think the shifts you saw were due to a neoplastic clone? Have you been able to phenotype the neoplasms, and do they fall within the clonal expansion or not?

\section{Miller:}

As to your specific question, lymphoma kills mice very quickly so that in cross-sectional pathology studies it is badly underestimated by factors of around three- to fourfold. In general, it is not recognizable to a pathologist, even working with sophisticated tools, a year prior to the demise of the mouse. That is the main reason we think it unlikely that the immune deficiency we saw was secondary to a malignant state. There was no statistically significant effect or detectable effect of $\mathrm{CD}^{+}, \mathrm{CD}^{+}$, or $\mathrm{CD}^{+}$numbers, so if these are clones, they are funny clones. They would have to be equally distributed among the $\mathrm{CD}^{+}, \mathrm{CD}^{+}, \mathrm{CD}^{+}$, and $\mathrm{B}$ cell lineage. That $I$ think argues against the idea that there was a clonal expansion of the malignant cell: it would have shown up as a change in $\mathrm{CD}^{+}$, $\mathrm{CD}^{+}, \mathrm{CD}^{+}$, or $\mathrm{B}$ cell numbers.

\section{Weksler:}

But you would agree that the neoplasm that killed them was clonal?

\section{Miller:}

Yes.

\section{Weksler:}

Did you see a clonal change?

\section{Miller:}

We did not look at the $\mathrm{T}$ cell receptor repertoire at 6 months of age. There was no change at 6 months in the mice that were destined to get $T$ cell tumors in $\mathrm{CD}^{+}$and $\mathrm{CD}^{+}$cells. The only changes we saw were those that were age sensitive in the memory virgin cell. 
As to your more general philosophical point, I am sensitive about the use of words to describe the age-associated changes. The reason $I$ am sensitive is that I have seen my grants, and grants of many other people, rejected by reviewers who argue that immune function aging is not a matter of deficiency, so why bother to study deficiency when it is due to autoantibodies? I think it is important in conveyi..1g to a casually educated public that the situation is more complex, and that it cannot be expressed in a single word.

\section{Keusch:}

To get back to the question of premalignancy: I wonder about your data with P-glycoproteins, since many glycoproteins and glycolipids are differentiation markers altered in the expression of neoplastic cells. Could it be a marker of ultradifferentiation, or a premalignant state?

\section{Miller:}

P-glycoprotein-positive cells increase with age in the mice we have studied. This could be an advantage to a cell and it may well be that the P-glycoprotein-high subsets of these cells are the ones destined to become malignant. We have no evidence for or against that idea. It is known that P-glycoprotein is high in tumor cells prior to exposure to antitumor drugs, so it is typically present at higher levels in malignant cells in ways that cannot be explained by xenotoxic selection. In all the old mice we looked at, about $70 \%$ of the $\mathrm{T}$ cells are high in P-glycoprotein, regardless of whether the mice later died of $T$ cell malignancies.

\section{Chandra:}

I am confused: first memory cells appear, then the malignancies appear, and then the malignancy contributes to the memory cells?

\section{Miller:}

Let me clarify that. There is a memory cell shift in some mice by 6 months of age; a year later these mice are found to have cancer. So we hypothesize that the mice who develop memory cells rather prematurely compared to their siblings are the ones who are predisposed to the developing cancer.

\section{Meydani:}

In the last few slides, were all the individual animals the ones that would later die? If not, how do you explain their survival?

\section{Miller:}

To develop a cancer you need to have more than a poor immune system-perhaps a genetic predisposition to that cancer or an encounter with a carcinogen. Cancer is a complicated phenomenon, and what we are trying to argue is that immune deficiency is not the only cause, but that it is one of the key factors that predispose to tumorigenesis. When all the mice have died it will be interesting to see whether those that had poor immunity at 6 months, 11 months, or 18 months, subsequently died of malignancies or a particular kind of malignancy.

\section{Kelley:}

Your data on the ability of memory cells to pump rhodamine out of the old mice was particularly interesting to me. You mentioned that you let it sit around for 30 minutes or an hour? Since $\mathrm{T}$ cells are now known to die apoptotically, is there any difference between the virgin cells and memory cells in terms of apoptosis in vitro?

\section{Miller:}

I don't know of any data on susceptibility of $\mathrm{T}$ cells from young or old mice to apoptosis. Rhodamine and calcium extrusion in these normal healthy cells is biochemically unrelated to apoptosis.

\section{Kelley:}

That is why I think it's a relevant question, but you have not looked at it?

\section{Miller:}

No we have not.

\section{Goodwin:}

Your data on malignancy, was it four or five or three or four mice that died of lymphoid malignancy? That is interesting. There is a lot of literature on immunosuppression in humans and subsequent malignancy and several explanations for these results come to mind. In humans, lymphoid malignancy is the tumor type that increases. If you just immunosuppress for something like rheumatoid arthritis, it is very difficult to show the increased malignancy. But in aging most of the increases could be considered lymphoid-type malignancies. Is immunosuppression in your model leading to the concept of immune surveillance? Or is the immunodysregulation that you are showing in these younger animals leading to immune-related tumors?

\section{Miller:}

The hypothesis I favor is that the premature decline in immunity and the premature development of disease are linked because they are both caused by changes in the rate of aging. We intend to study these mice from many aspects of immune and nonimmune aging. We have collaborators who will be measuring fibroblast growth rate, IL-6 production, collagen cross-linking, retroviral production, and skeletal muscle function. If many or all of these parameters decline in parallel in those mice that die young, that will make a strong case for their being linked together, not by cause and effect mechanisms but because they are associated with relatively pre- 
mature aging. It would be hard to argue that loss of muscle strength is what leads to lymphoma. If, however, we find that the only good predictors of neoplasm are the immune markers and that the retrovirus or muscle strength and collagen had no correlation, then the hypothesis you just suggested, that immune deficiency specifically predisposes an individual to immune neoplasms, will be supported. Some of our animals are now dying of other diseases, including amyloid, aortic aneurysms, and nephritis. The mice are genetically heterogeneous, and fortunately they are dying of many different things, which we can now examine in this context.

\section{Keusch:}

It has recently been demonstrated that some of the differentiation markers related to glycolipids are signals for apoptosis. If the P-glycoprotein changes, or if there is some alteration in terminal differentiation, that might pertain to other glycosylated surface markers that may be signals for apoptosis and loss of populations.

\section{Miller:}

We are not at all convinced that the increase with age in P-glycoprotein function accounts for the important properties of these cells. We think it is more likely to be a marker of some other differentiation state that was not well tracked before the rhodamine-extrusion test. Cells high in P-glycoprotein may differ in interesting ways. IL-4 production in the presence of IL-2 is the first for which we have good data, but there may well be other sensitivities, which predispose them to autoreactivity. We need to look at all of this.

\section{Weindruch:}

I think your hybrid mice are an excellent model. I have always been surprised at the amount of heterogeneity that I see in inbred strains, not only with immunologic assays but also with very basic things like metabolism efficiency. Some mice eat a lot of food and do not weigh much; heavier mice eat less food. Have you done statistical analyses in your hybrid mice for the degree of variation in markers of immune senescence you measured, and have you compared that to the old "standard" brand of mice?

\section{Miller:}

Once we have a larger data set on these hybrid mice we will be asking those questions. Drs. Jay Phelan and Steve Austard have asked the more general question: do inbred mice have a narrower or wider variance compared to genetically heterogeneous animals? Based on a literature survey, they have shown that inbreeding often does not lead to a narrowing of variance, but rather to an expansion in phenotypic variance; the control mechanisms that we heterozygotes maintain for homeostatic control have been eliminated in inbred animals. Therefore, we need to test that specific question. My expectation is there will not be a great increase in variance in the hybrid mice.

\section{Chandra:}

What proportion of the old mice die of diseases other than tumors, and do you have any preliminary information on lymphocyte subset changes in those?

\section{Miller:}

The 48th percentile mice died earlier this week, so as yet we have no information on the genetic background of the last half of the aging curve. Lymphomas continue to predominate. More than half of the mice die with some form of malignancy, but we also have seen animals that have died of hemangiosarcoma, granulocytic leukemia, aortic or vascular aneurysm, and nephritis. We are still waiting to see what will happen to the remaining $50 \%$ of the population.

\section{Chandra:}

The majority die of tumors, is that true?

\section{Miller:}

Yes.

\section{Weindruch:}

If we feed a normal-calorie diet to our strains of $F_{1}$ and C57BL/6, we see about $80 \%$ dying with cancer; typically lymphoma and hepatoma. If we feed low-calorie diets, cancer incidence is reduced $30-40 \%$.

\section{Bendich:}

Is there a virus that causes tumors in mice?

\section{Miller:}

If mice are homozygous for a virus that causes thymic lymphoma, and also have genes that make them susceptible, and you make an $F_{1}$ hybrid, the incidence of the AKR type tumor goes down. The AKR genotype is randomly distributed across these animals so that the animal has $25 \%$ of the AKR genotype.

\section{Bendich:}

Do you look for the virus?

\section{Miller:}

We looked for the pathology caused by the virus, and we did not see it in these animals. There are also other tumor viruses, segregating in these crosses, but the heterozygosity tends to protect them from diseases typically present in inbreds.

\section{Weksler:}

In William Adler's report from The Baltimore Longitudinal Study on Aging, there was an unexplained decrease in lymphocyte counts in the 3 years prior to a person's demise from any cause. 


\section{Miller:}

A $10-15 \%$ decline in total lymphocyte number occurs in the year prior to death in healthy humans, regardless of the age of death.

\section{Chandra:}

This indication grows stronger if there is also cutaneous anergy to delayed hypersensitivity antigens.

1. Flurkey K, Stadecker M, Miller RA. Memory T lymphocyte hyporesponsiveness to non-cognate stimuli: a key factor in age-related immunodeficiency. Eur $\mathrm{J}$ Immunol 1992;22:931-5

2. Philosophe B, Miller RA. Diminished calcium signal generation in subsets of $\mathrm{T}$ lymphocytes that predominate in old mice. J Gerontol 1992;57:B87-93

3. Witkowski JM, Li SP, Gorgas G, Miller RA. Extrusion of the P-glycoprotein substrate rhodamine-123 distinguishes CD4 memory $T$ cell subsets that differ in IL-2 driven IL-4 production. J Immunol 1994;153: 658-65

4. Miller RA, Turke C, Ruger J, et al. Age-sensitive T cell phenotypes covary in genetically heterogeneous mice and predict early death from lymphoma. J Gerontol, in press

5. Lerner A, Yamada T, Miller RA. Pgp-1hi T lymphocytes accumulate with age in mice and respond poorly to concanavalin A. Eur J Immunol 1989;19:977-82

6. Miller RA, Jacobson B, Weil G, Simon ER. Diminished calcium influx in lectin-stimulated $T$ cells from old mice. J Cell Physiol 1987;132:337-42

7. Shi J, Miller RA. Tyrosine-specific protein phosphorylation in response to anti-CD3 antibody is diminished in old mice. J Gerontol 1992;47:B147-53

8. Philosophe B, Miller RA. T Iymphocyte heterogeneity in old and young mice: functional defects in $T$ cells selected for poor calcium signal generation. Eur $\mathrm{J} \mathrm{Im-}$ munol 1989;19:695-9

9. Shi J, Miller RA. Differential tyrosine-specific protein phosphorylation in mouse $T$ lymphocyte subsets. Effect of age. J Immunol 1993;151:730-9

10. Witkowski JM, Miller RA. Increased function of P-glycoprotein in $T$ lymphocyte subsets of aging mice. $J$ Immunol 1993;150:1296-306

11. Li SP, Miller RA. Age-associated decline in IL-4 production by murine $T$ lymphocytes in extended culture. Cell Immunol 1993;151:187-95 